\title{
The Determination of Paleo-Intensities of the Earth's Magnetic Field with Emphasis on Mechanisms which Could Cause Non-ideal Behavior in Thellier's Method
}

\author{
Robert S. COE* \\ Department of Geology and Geophysics, University of California, Berkeley, California.
}

\begin{abstract}
95 NRM-TRM curves were determined by Thellier's method from a variety of volcanic rocks. Most of them deviate from a straight line over parts of their length, sometimes so much that not even a crude estimate of paleo-intensity can be made. Some of the many possible causes of such non-ideal behavior include the effects of the sample demagnetizing field, secondary components of magnetization, mechanisms of acquisition of TRM which violate the assumptions of Thellier's method (such as nonlinearity of TRM with field), changes in the TRM spectrum induced by heating in the laboratory, and others. Where possible these mechanisms are discussed from both a theoretical and experimental standpoint, and their effects are identified in the NRM-TRM curves. In addition, diagnostic tests designed to determine quickly the suitability of a rock for intensity studies were sought. Tests tried included the comparison of heating and cooling $J_{s}-T$ curves, measurement of susceptibility before and after heating, and others. None were adequate. Finally, the quicker method of simply comparing the NRM and the total TRM is compared with Thellier's method for determining paleo-intensities. The latter is clearly the more informative and reliable when dealing with individual units, but the former may be useful for deriving average values of the paleo-intensity during geologic periods from large suites of volcanic rocks of varying types.
\end{abstract}

\section{Introduction}

In a previous paper (Coe, 1967) paleo-intensities of the geomagnetic field derived from Tertiary and Quaternary volcanic rocks by Thellier's method (Thellier and Thellier, 1959) were given. The purpose of this paper is to discuss both with respect to the experimental data and existing theory some of the factors which limit or completely prevent the successful application of Thellier's method. Until a much greater understanding in this area is attained it will continue to be difficult to assess with any confidence the extent of systematic errors in the determintion of paleo-intensities.

In Thellier's method a linear relation is assumed to exist between the natural remanent magnetization (NRM or $D_{n}\left(T_{r}, T\right)$ ) remaining after heating to an elevated temperature $T$ and cooling to room temperature $T_{r}$ in zero magnetic field and the artificial thermoremanent magnetization (TRM or $J_{a}\left(T, T_{r}\right)$ ) produced by cooling the same sample from $T$ to $T_{r}$ in

* Currently at the Department of Geophysics, Australian National University, Canberra, A.C.T., Australia until March, 1968; at the University of California, Santa Cruz, California thereafter. 
an applied magnetic field $F_{a}$. This relation is expressed analytically by

$$
D_{n}\left(T_{r}, T\right)=J_{N}-\left(F_{e} / F_{a}\right) J_{a}\left(T, T_{r}\right)
$$

and graphically by Fig. 1. $J_{N}$ is the original NRM before any demagnetization, and $F_{e}$ is the paleo-intensity, i.e., the strength of the field which produced $J_{N}$. This system of expressing the results was first proposed by Arai (1963, M.Sc. thesis, University of Tokyo) and was first published by Nagata, Arai and Momose (1963). Thellier's method, as applied in this study, is discussed more fully in the previous paper (Coe, 1967).
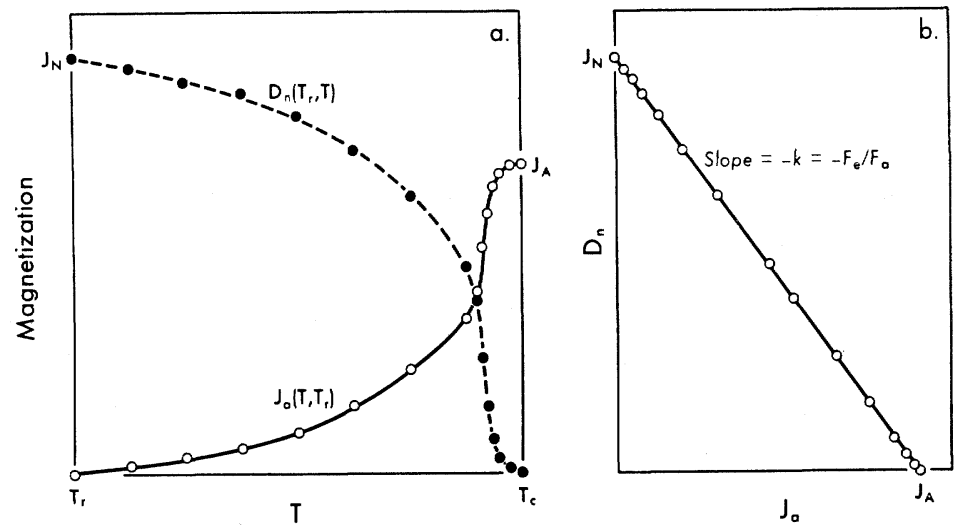

Fig. 1 (a) A hypothetical example of an ideal NRM demagnetization curve, $D_{n}\left(T_{r}, T\right)$, and an ideal TRM acquisition curve, $J_{a}\left(T, T_{r}\right)$.

(b) The corresponding ideal NRM-TRM curve after elimination of $T$-a straight line.

The ideal linear relation (1) requires that several conditions be met. (i) The magnetic field inside the sample must equal the actual paleo-intensity, $F_{e}$. (ii) The NRM must remain completely unchanged for all time after the rock has cooled, so that it is still identical with the original TRM. (iii) The partial thermoremanent magnetization (PTRM) acquired in every temperature interval must be uniquely associated with that interval, independent of the state of magnetization of the rock and the rate of cooling, and linear with applied field. (iv) The PTRM spectrum (the distribution of TRM with temperature) must remain unchanged during the heating experiments in the laboratory.

In this study ninety-five NRM-TRM curves were determined in Thellier experiments. The majority of them deviated systematically from a straight line over some temperature interval. The ultimate problem is to identify which non-ideal mechanisms are operative in each sample from the shape of the NRM-TRM curve and to evaluate their effects so that a reliable paleo-intensity can still be obtained. At present such a fundamental treatment is not possible; the gaps in both theoretical and experimental knowledge are too great. This paper attempts a modest beginning in that direction.

\section{Mechanisms Causing Deviations from Ideal Behavior}

Sample demagnetizing field. Every magnetized body, as a direct result of its magnetization, lexperiences an internal self-demagnetizing field $\boldsymbol{H}_{d}$. Thus the internal field $\boldsymbol{H}_{i}$ differs 
from the externally applied field $\boldsymbol{H}$ :

$$
\boldsymbol{H}_{i}=\boldsymbol{H}+\boldsymbol{H}_{a}
$$

If the body is an ellipsoid with uniform magnetization $\boldsymbol{J}_{T}$ directed along one of the principal axes, it can be shown that

$$
\boldsymbol{H}_{d}=-N \boldsymbol{J}_{T}
$$

at all points inside (Stoner, 1945), where $N$ is a constant known as the demagnetizing factor. In what follows it is assumed that the actual rock sample approximates this model.

There are two levels in which a demagnetizing field appears in a rock: within each magnetic grain (interaction of the grain on itself) and in the less intensely magnetized sample as a whole (interaction of the grains on each other.) It is only the latter effect which is considered here. In a strongly magnetic rock the ideal linearity of the NRM-TRM curve will be lost because the internal field which produces the magnetization also depends upon the magnetization.

The actual shape of the curve will depend on many things: (i) the magnitude of the earth's field $F_{e}$ and the demagnetizing factor $N_{n}$ existing when the rock unit acquired its NRM by cooling originally, (ii) the magnitude of the NRM $J_{N}$ thereby produced, (iii) the magnitude of the artificial field $F_{a}$ and the demagnetizing factor $N_{a}$ of the sample studied in the lab (in my experiments $0.452 \leqslant F_{a} \leqslant 0.834 œ$ and $N_{a} \cong \frac{4}{3} \pi$ ), (iv) the particular procedure used in the Thellier experiments, and (v) the mode of acquisition of TRM by the sample. Of this list least is known about (v), and there may be considerable variation in the mechanism of formation of TRM from sample to sample or even more than one mechanism within the same sample.

Let us assume that no matter what the specific mechanism is, when a sample with only one species of magnetic mineral cools an amount $d T$ in an internal field $\boldsymbol{H}_{i}$, the increase in remanence $d \boldsymbol{J}$ is given by

$$
d J=H_{i} K(T) d T+\frac{1}{J_{s}} \frac{d J_{s}}{d T} J d T
$$

where $d \boldsymbol{J}, \boldsymbol{J}$, and $\boldsymbol{H}_{i}$ are assumed to be parallel. The second term describes the increase of a preexisting remanence $J$ due to the increase of $J_{s}(T)$, the spontaneous magnetization. The first term specifies the acquisition of new remanence in terms of the internal field and a function $K(T)$ characteristic of the particular sample.

The internal field depends on the total magnetization $\boldsymbol{J}_{T}$, which is equal to the remanent $(\boldsymbol{J})$ plus induced $\left(\boldsymbol{I}=X(T) \boldsymbol{H}_{i}\right)$-i.e.,

$$
\boldsymbol{J}_{T}=\boldsymbol{J}+X(T) \boldsymbol{H}_{i}
$$

Assuming $\boldsymbol{H}$ is parallel to $\boldsymbol{J},(2),(3)$ and (5) show that $\boldsymbol{H}_{\boldsymbol{i}}$ is parallel to $\boldsymbol{H}$ and given by

$$
H_{i}=\frac{H-N J}{1+N X}
$$

Substitution of (6) into (4) yields a linear first order differential equation whose general solu- 
tion is

$$
J(T)=\left[J_{s}(T) \exp \left\{-\int N P(T) d T\right\}\right]\left[H \int \frac{1}{J_{s}(T)} \exp \left\{\int N P(T) d T\right\} P(T) d T+C\right]
$$

where $P(T)=\frac{K(T)}{1+N X(T)}$ and $C$ is a constant determined by the initial conditions. This solution is not of much practical use unless everything is known about the sample.

The problem, however, can be approached in another way. Instead of seeking as in (4) to describe the remanence at all temperatures $T$ during cooling, let us refer it to a lower temperature $T^{\prime}$ at which all measurements are made. Thus cooling from $T+d T$ to $T$ in a field $H$ produces an increase in remanence $d J^{\prime}$ (measured at the lower temperature $T^{\prime}$ ) which is given by

$$
d J^{\prime}=H_{i} S(T) d T
$$

where

$$
S(T)=\frac{J_{s}\left(T^{\prime}\right)}{J_{s}(T)} K(T)
$$

For most rocks $N X \leqslant 0.01$ (Nagata, 1961, p. 128 and 143), so that to a good approximation (6) can be written

$$
H_{i}=H-N J
$$

The $J$ of (8) is the remanence at $T \geqslant T^{\prime} . J_{s}$ increases with decreasing temperature, so that replacing $J$ in (8) by its value referred to $T^{\prime}, J^{\prime}$, sets an upper limit to the disturbing effects of the demagnetizing field, because $J^{\prime} \geqslant J$. When substituted in (7), this approximation leads to an equation which is possible to work with:

$$
\frac{d J^{\prime}}{H-N J^{\prime}}=S(T) d T
$$

which is only exactly true when $J_{s}(T)$ is a constant at all temperatures below the maximum temperature at which new TRM is acquired.

The solution is

$$
\int_{J_{1}}^{J_{2}} \frac{d J^{\prime}}{H-N J^{\prime}}=\frac{1}{N} \ln \frac{H-N J_{1}}{H-N J_{2}}=\int_{T_{1}}^{T_{2}} S(T) d T,
$$

where $J_{1}$ is the initial remanence measured at $T^{\prime}$, but acquired above $T_{1}$, and $J_{2}$ is the final remanence measured at $T^{\prime}$ after cooling from $T_{1}$ to $T_{2}$ in an applied field $H$. This general result can now applied to the Thellier experiments, in which the measurements were made at room temperature $T^{\prime}=T_{r}$.

When the sample cools in the natural state from above the Curie temperature $T_{c}$, to $T \geqslant T_{r}$ it acquires an NRM, in the earth's magnetic field which, if it were measured at $T_{r}$, would be $J_{n}(T)$. As cooling is completed and the temperature becomes constant at $T_{r}$, the NRM reaches its final value $J_{N}=J_{n}\left(T_{r}\right)$. Then (10) yields

$$
\frac{1}{N_{n}} \ln \frac{F_{e}-N_{n} J_{n}}{F_{\bullet}-N_{n} J_{N}}=\int_{T}^{T_{r}} S(T) d T .
$$


The sample is later removed from the lava, thereby changing its demagnetizing factor from $N_{n}$ to $N_{a}$, and studied in the lab. First it is heated from $T_{r}$ to $T$ and cooled from $T$ to $T_{r}$ in zero applied field. The remaining NRM $D_{n}(T)$ is not equal to $J_{n}(T)$ because of the TRM added by the sample demagnetizing field. Using (10) again, it follows that

$$
\frac{1}{N_{a}} \ln \frac{J_{n}}{D_{n}}=\int_{T}^{T_{r}} S(T) d T \text {. }
$$

Next the sample is reheated to $T$ and cooled to $T_{r}$ in a field $F_{a}$, adding a component of TRM $J_{a}(T)$ such that the final value of magnetization is $J(T)=J_{n}(T)+J_{a}(T)$. Only the two extreme cases are considesed: (a) $F_{a}$ parallel to the NRM, $J=J_{n}+J_{a}$, and (b) $F_{a}$ antiparallel to the $\mathrm{NRM}, J=J_{n}-J_{a}, J_{a}$ taken as greater then zero in both instances. Applying (10),

$$
\begin{aligned}
& \frac{1}{N_{a}} \ln \frac{F_{a}-N_{a} J_{a}}{F_{a}-N_{a}\left(J_{n}+J_{a}\right)}=\int_{T}^{T_{r}} S(T) d T \\
& \frac{1}{N_{a}} \ln \frac{F_{a}+N_{a} J_{n}}{F_{a}+N_{a}\left(J_{n}-J_{a}\right)}=\int_{T}^{T_{r}} S(T) d T
\end{aligned}
$$

$S(T)$ is the same function in all the equations. Hence (11) with (13a) or (13b) gives

or

$$
\begin{aligned}
& J_{a}=\left(\frac{F_{a}-N_{a} J_{n}}{N_{a}}\right)\left\{1-\left(\frac{F_{e}-N_{n} J_{N}}{F_{e}-N_{n} J_{n}}\right)^{N_{a} / N_{n}}\right\} \\
& J_{a}=\left(\frac{F_{a}+N_{a} J_{n}}{N_{a}}\right)\left\{1-\left(\frac{F_{e}-N_{n} J_{N}}{F_{e}-N_{n} J_{n}}\right)^{N_{a} / N_{n}}\right\}
\end{aligned}
$$

Combining (12) with either (13a) or (13b) and then with (14a) or (14b) yields the same result for $D_{n}$, namely

$$
D_{n}=J_{n}\left(\frac{F_{e}-N_{n} J_{N}}{F_{e}-N_{n} J_{n}}\right)^{N_{a} / N_{n}}
$$

(15) and either (14a) or (14b) specify the NRM-TRM curve $\left(D_{n}-J_{a}\right.$ curve) in terms of the parameter $J_{n}$, which decreases monotonically from $J_{N}$ to 0 as the temperature $T$ of each step of the Thellier experiment increases from $T_{r}$ to $T_{c}$. The detailed dependence of $J_{n}$ on $T$ varies from sample to sample, yet these results are independent of the particular sample in this respect because $T$ does not appear explicitly in (15) and (14a) or (14b).

In the limit of a weakly magnetic sample this solution reduces to the ideal case. That is, when $N J_{N} / F \ll 1$, where $N$ is the larger of $N_{n}$ or $N_{a}$ and $F$ is the smaller of $F_{e}$ or $F_{a}$, expansion of (14a) or (14b) and (15) and dropping of all but first order terms yields the ideal relation (1).

Let us suppose that an apparent paleo-intensity is inferred by connecting the initial and final points of the non-ideal (curved) NRM-TRM curve. To a good approximation the demagnetizing factor $N_{a}$ of the laboratory samples can be taken as $\frac{4}{3} \pi$, whereas for the samples in their natural state $N_{n}$ may take on any value between 0 and $4 \pi$. A typical mag. netic sample might acquire an NRM of $3 \times 10^{-3} \mathrm{emu} / \mathrm{cm}^{3}$ in a field of $0.5 œ$, that is, it is characterized by a ratio of $J_{N} / F_{e}=0.006$. For such a sample (14a) or (14b) and (15) show that the apparent paleo-intensity might differ from the true paleo-intensity by as much as $+1.3 \%$ 


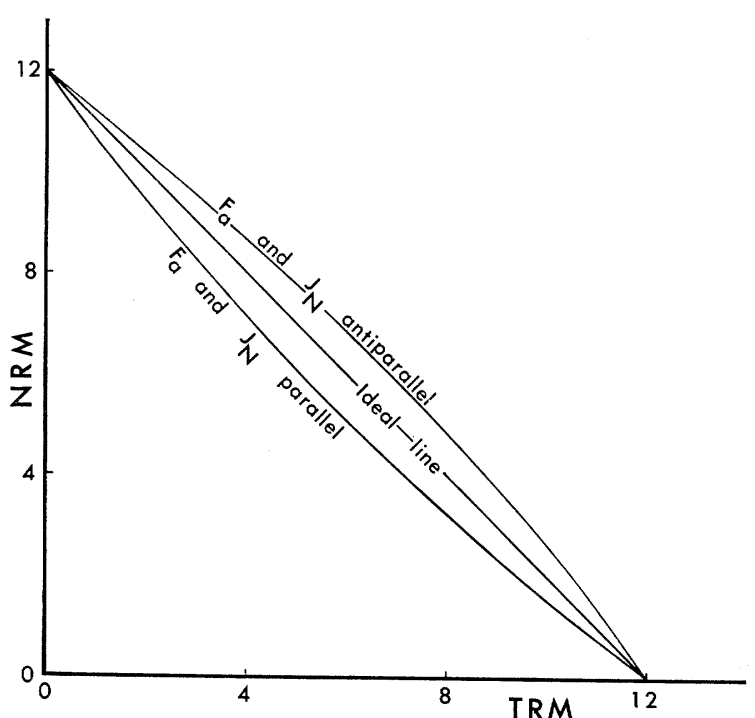

Fig. 2 The effect of the sample demagnetizing field on the NRM-TRM curve as predicted by equations $14 \mathrm{a}, 14 \mathrm{~b}$ and 15 when $N_{n}=N_{a}=\frac{4}{3} \pi, F_{e}=F_{a}$ and $J_{N} / F_{e}=0.06$. (Axes are labelled in units of $10^{-3}$ $\left.\mathrm{emu} / \mathrm{cm}^{3}\right)$. or $-2.5 \%$ when $N_{n}$ is 0 or $4 \pi$, respectively, whereas they are equal when $N_{n}=\frac{4}{3} \pi$. In all three cases the curvature of the NRM-TRM curve is negligible. In rather strongly magnetic rocks, however, where $J_{N} / F_{e}=0.06$, the comparable errors predicted by the formulas range from $+15 \%$ to $-33 \%$. Moreover, the curvature is not negligible, as is illustrated by the two curves in Fig. 2 for the case $N_{n}=\frac{4}{3} \pi$.

The predictions of formulas (14a), (14b) and (15) have not been sufficiently tested by experiment. A few experiments of a preliminary nature were conducted in which several samples were given total TRM's in the lab and then subjected to the routine experiments of Thellier's method $\left(F_{a}=F_{e}=\right.$ $\left.0.45 œ, N_{a}=N_{n}=\frac{4}{3} \pi\right)$. These showed that the actual NRM-TRM curve is often more complex than predicted. The curvature is also usually of lesser degree than that predicted by formula, and sometimes even of the wrong sign.

These discrepancies could be due to inadequacies in the treatment which has been presented of the sample demagnetizing field or to competing non-ideal mechanisms which have not been considered. I believe that the results do provide valid estimates of the maximum error in the determination of paleo-intensities that could possibly arise from the effects of the sample demagnetizing field.

Secondary components of magnetization. The original NRM is often masked to some degree by secondary components of magnetization acquired after cooling of the rock. Samples containing significant isothermal remanent magnetization (IRM) due to nearby lightning strikes usually show scattered directions and characteristic ac demagnetization curves (Cox, 1961) and can be avoided in intensity studies. Chemical remanent magnetization (CRM) is more difficult to detect, but may not be very important in young volcanic rocks which are fresh in general appearance. Viscous remanent magnetization (VRM) exists to some extent in most samples, and when significantly large can be best identified by the shape of the NRM-TRM curve, as will be discussed shortly.

An attempt was made to combine ac demagnetization with Thellier's method in order to make available for intensity studies samples with large secondary components. Four pairs of samples which were essentially free of secondary magnetization were studied, the members of each pair being adjacent samples from the same core. The first member was 


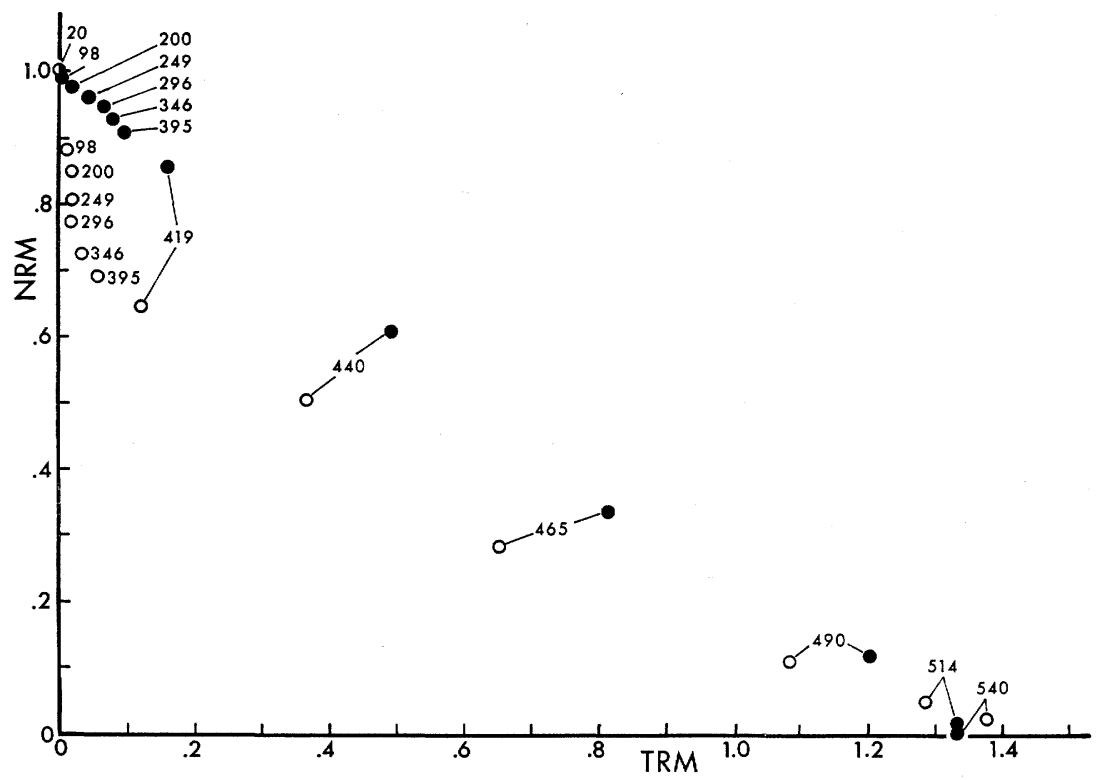

Fig. 3 Comparison of normalized NRM-TRM curves of samples of basalt from the same core. Solid circles: the sample was used in the standard Thellier experiments (normalizing factor $=32.3 \times 10^{-3} \mathrm{e} \mathrm{mu} / \mathrm{cm}^{3}$ ). Open circles : the sample was partially demagnetized in an alternating field of 200 œ before each measurement of magnetization in the Thellier experiments (normalizing factor $\left.=16.6 \times 10^{-3} \mathrm{emu} / \mathrm{cm}^{3}\right) . \quad F_{a}=0.834 \propto$. The numbers associated with the points are the temperatures in ${ }^{\circ} \mathrm{C}$ of the heating steps.

tested in the standard Thellier's method, while the second was partially demagnetized in an alternating field of 100 or 200 œ prior to the heating experiments and also after each application of PTRM in order that the magnetization measured at each stage would be commensurate with the initial "cleaned" NRM.

One pair of samples produced straight lines with identical slopes, indicating that the technique is suitable for this rock, but the other three pairs of curves differ markedly in shape. For those three cores the curves of samples which received ac demagnetization are more concave upward than those of the untreated specimens, as shown, for example, by the pair of curves of Fig. 3.

The ac demagnetization appears to have caused increased removal of NRM and decreased acquisition of TRM at the lower temperatures. It is strange and perhaps significant, however, that the slopes of the lines connecting the endpoints of both curves of each pair are equal within 5 to $7 \%$ in all four cases. This point will be discussed later.

These findings are preliminary; more samples from different volcanic units should be tested. Nonetheless, the suggestion at present is that the method will probably not be a suitable one for paleo-intensity studies. Thermal demagnetization, which is an integral part of Thellier's method, appears much more effective in passively removing the effects of secondary components, for in many samples the NRM is all original TRM except for a few percent with low blocking temperatures. When other disturbing factors are absent, the 
higher temperature portion of the NRM-TRM curve can be used to determine the paleointensity.

$V i s c o u s$ remanent magnetization (VRM) and spontaneous decay: single-domain grains. The only theory of TRM which is on a firm enough basis to permit order of magnitude calculations of magnetic stability over millions of years is the theory for single-domain grains (Néel, 1949). This theory, however, may also account for the acquisition of TRM -or at least a part of the TRM - by multidomain grains in rocks (Verhoogen, 1959; Ozima and Ozima, 1965 ; Stacey, 1967).

Néel (1949, p. 109-114) shows that the magnetization of a diffuse assemblage of identical single-domain grains of uniaxial anisotropy aligned parallel to each other will decay to $1 / e$ of its value in zero external field within a time $\tau_{0}$,

where

$$
\tau_{0}=\frac{1}{C} \exp \frac{v H_{c} J_{s}}{2 k T}
$$

and

$$
C=\frac{e H_{c}}{m}\left\{3 G \lambda+D J_{s}^{2}\right\}\left(\frac{2 v}{\pi G K T}\right)^{\frac{1}{2}}
$$

Using values of the constants appropriate for magnetite at room temperature $\left(J_{s}=480 \mathrm{emu} /\right.$ $\mathrm{cm}^{3}, G=$ shear modulus $=10^{12}$ dynes $/ \mathrm{cm}^{2}, \lambda=$ magnetostriction constant $=\frac{2 \lambda_{100}+3 \lambda_{111}}{5}=$ $38 \times 10^{-6}, k=$ Boltzmann's constant $=1.38 \times 10^{-16} \mathrm{erg} / \mathrm{deg}, e / m=$ ratio of charge to mass of an electron $=1.76 \times 10^{-7} \mathrm{emu} / \mathrm{g}$, and $D=$ shape factor of grain $=3$ ), it follows that $\tau_{0} \geqslant 3 \times 10^{9} \mathrm{yr}$ if the grain coercivity $H_{c} \geqslant 10 œ$ and the grain volume $v \geqslant 120 \times 10^{-18} \mathrm{~cm}^{3}$. This volume implies a mean grain diameter of about 0.05 micron, which is less than the maximum diameter above which single-domain grains of magnetite cannot exist (about 0.1 micron).

If the TRM is opposed by a weak magnetic field $h$, the relaxation times are not drastically altered (Néel, 1949, p. 115-116). With the same values of the parameters as before, it can be shown that when $h=0.5 œ$ the relaxation time is $\tau \geqslant 1.5 \times 10^{9} \mathrm{yr}$, and when $h=2.5 œ$ $\tau \geqslant 4 \times 10^{8} \mathrm{yr}$. Thus it is at least suggestive that much of the TRM of volcanic rocks may be very stable with respect to time, because demagnetization of samples in alternating fields shows that usually $H_{c}>10 œ$. Such calculations, however, cannot be applied to spceific samples because of the practical impossibility of determining $v, H_{c}$ and $J_{s}$ for each grain or region of a grain which behaves as a single domain.

Nonetheless, much insight into the actual behavior of rocks can be gained by the qualitative application of Néel's theory. Suppose a rock consisting of an assemblage of single-domain grains of uniaxial anisotropy with a distribution of volumes $v$ and coercivities $H_{c}$ acquires a TRM by cooling in a weak magnetic field $h$. If $h$ remains constant in magnitude and direction, how will the magnetization change during the course of unlimited time?

The potential energy lost by a grain when its magnetization rotates from antiparallel to parallel to $h$ is

$$
\Delta E=2 v h J_{s}(T)
$$

where $J_{s}(T)$, the spontaneous magnetization, increases with decreasing temperature $T$. The 


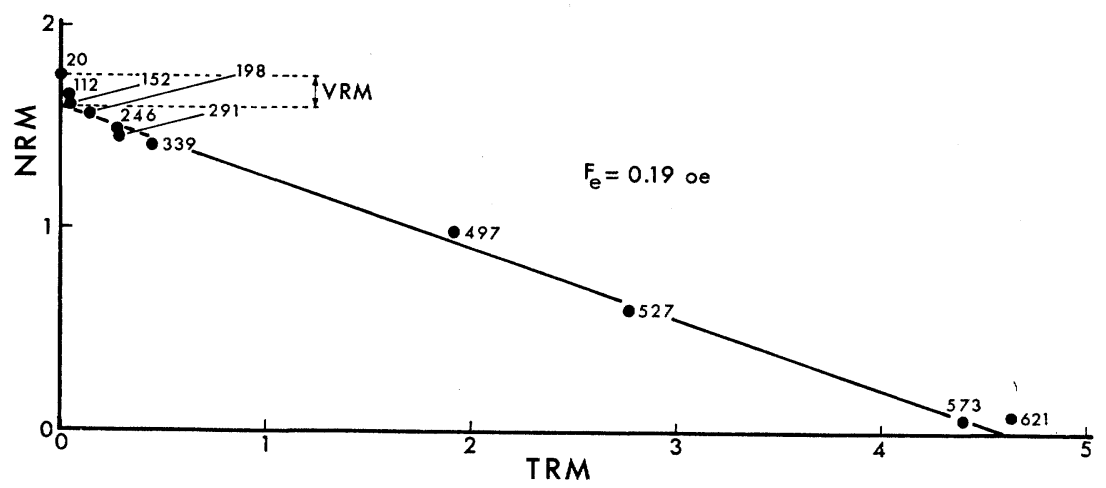

Fig. 4 The effect of VRM on the NRM-TRM curve of a sample which acquired its NRM in a normal field of paleo-intensity considerably less than average (see text). This sample is of Pleistocene Crater Lake andesite (Coe, 1967, table 1). $F_{a}=0.534 œ$. The axes are labelled in units of $10^{-3} \mathrm{emu} / \mathrm{cm}^{3}$, and the numbers associated with the points are the temperatures in ${ }^{\circ} \mathrm{C}$ of the heating steps.

equilibrium ratio of grains in the parallel state to those in the antiparallel state at any given $T$ is determined by the ratio of $\Delta E$ to the thermal energy $k T$ according to the Boltzmann distribution $\exp (-\Delta E / k T)$. As cooling progresses, the equilibrium ratio changes. For a given cooling rate, there exists a narrow range of temperatures characteristic of each grain ("the blocking temperature," $\mathrm{T}_{b}$ ) below which thermal agitation is not sufficient to maintain equilibrium. In a sufficiently long time, though, equilibrium will eventually be regained because of thermal fluctuations, and the magnetization residing in grains with $T_{b}$ significantly above the ambient temperature will increase. The rate of increase for each ensemble of identical grains of the whole assemblage will be determined by the degree of disequilibrium and the relaxation time.

Thus under conditions of constant geomagnetic field the TRM of a rock will not decay, nor will the VRM be concentrated in those grains with low blocking temperatures (short relaxation times). Instead, the grains with low $T_{b}$ will be least affected, those with intermediate $T_{b}$ will acquire a VRM first, and those with high $T_{b}$ will acquire a VRM more slowly which in the long run will proportionally exceed the VRM in the other grains. The NRM-TRM curve of a sample which had proceeded part, but not all, of the way to equilibrium would be $S$-shaped: concave down at low temperatures and concave up at high temperatures.

The earth's magnetic field, however, has not remained constant. Within the past ten thousand years the intensity has probably varied by at least a factor of three or four (Thellier and Thellier, 1959; see Coe, 1967, for additional primary references), and within the past few million years has actually reversed directions several times (Cox et al., 1964 and 1966; Doell and Dalrymple, 1966). This complex behavior makes it difficult to predict the actual VRM even for a sample of known age containing only single-domain grains; however, some qualitative statements can be made.

If such a sample acquired a TRM during the present normal polarity epoch, and if the field at that moment were significantly less than the average value during the ensuing ti me 


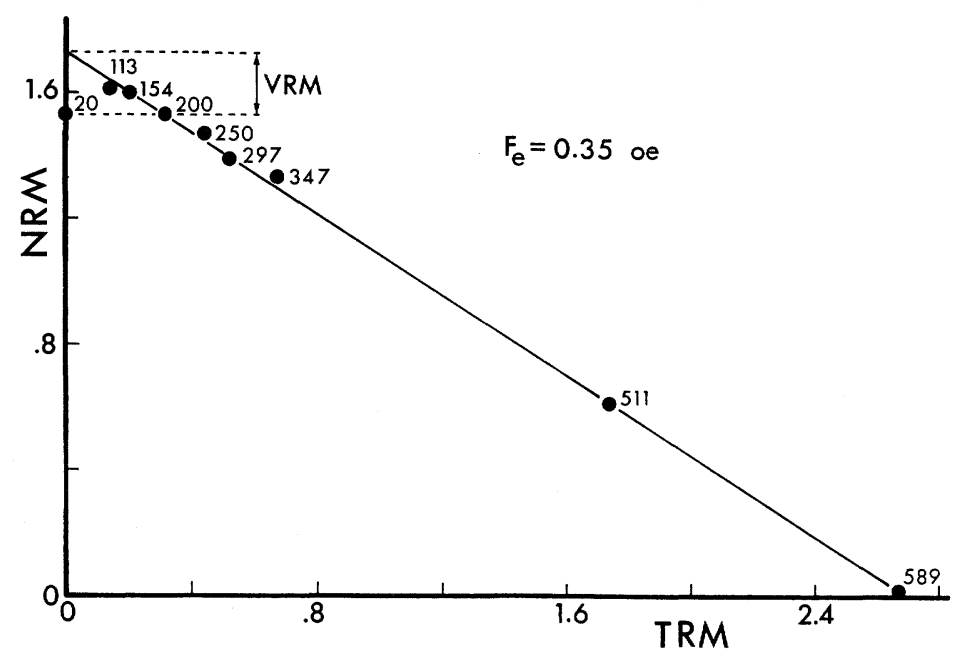

Fig. 5 The effect of VRM on the NRM-TRM curve of a sample which acquired its NRM in a reversed field. This sample is a sediment baked by a basalt flow in the Pliocene or Pleistocene from along the Airport Rd. near Alturas, Calif. (Coe, 1967, table 1). $F_{a}=0.534$ $œ$. The figure is labelled in the same way as figure 4 .

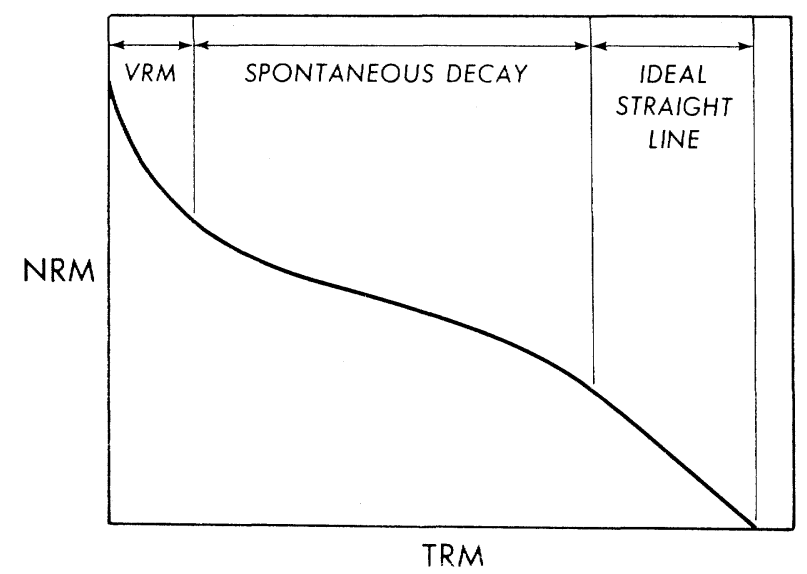

Fig. 6 A possible form of the NRM-TRM curve of a hypothetical sample which has acquired VRM in low blocking temperature domains and has undergone spontaneous decay in higher blocking temperature domains.

up to the present, then a VRM would be added parallel to the original TRM. Because $h$ is not constant, the grains with low $T_{b}$ would also be out of equilibrium (16), the VRM would first be manifested in these grains with shorter relaxation times, and the NRM-TRM curve would be concave up. Several samples which are probably from the present polarity epoch and which indicate low paleo-intensities display this behavior, and one example is given in Fig. 4.

Conversely, if the TRM-producing field were greater than average, a segment of the 
original TRM with low $T_{B}$ would decay while the remaining TRM with higher $T_{B}$ would increase. Depending on the magnitude of the decrease of the later ambient field and on the $T_{B}$ spectrum of the rock, the total TRM might monotonically increase, monotonically decrease or first decrease and then increase with time. Thus even in this simplified analysis, where only two states of magnetization are assumed possible for each grain-parallel or antiparallel to the field, the distinction between decay of original TRM and addition of VRM antiparallel to the original TRM becomes arbitrary and somewhat misleading. It is quite possible for the total TRM to decrease even though the ambient field is still parallel to it, and in this case the greatest portion of the NRM-TRM curve would be concave down. No examples of this behavior were discovered in the normally magnetized rocks studied. When the field reverses, howerer, the (algebraic) decrease in intensity is large, and eleven out of the twelve reversed samples studied exhibit NRM-TRM curves which are initially concave down (Fig. 5).

The history of the polarity changes of the geomagnetic field shows that the intensity averaged vectorially over the last several million years is probably much closer to zero than a typical instantaneous value. If this is so, then in rocks several million years of age and older the NRM which has relaxation times substantially greater than $10^{6}$ years must have undergone some spontaneous decay. (The NRM with relaxation times less than $10^{6}$ years will be affected mostly by the field of the last polarity epoch). Assuming such a sample to have a continuous spectrum of magnetization with relaxation times ranging from much less to much greater than $10^{6}$ years and to have acquired its original TRM in a normal field, the NRM-TRM curve might look like that of Fig. 6: initially concave up and finally concave down. No samples which are several million years old were studied which convincingly display this effect of spontaneous decay, but it may be discovered when more systematic work is done on older, magnetically soft rocks.

Attempts have been made to determine the susceptibility of samples to VRM by direct experiments. Extrapolation of the results to longer times is accomplished with the relation $\mathrm{VRM}=\mathrm{Slog}$, where $t$ is the time and $S$ is the magnetic viscosity constant. (See, for example, Nagata, 1961, p. 247). Shimizu (1960) used this method on samples of powdered magnetite, and states that the VRM acquired in a field of $3.3 œ$ would equal half the TRM acquired in the same field only after the passage of $10^{120}$ years. This result, however, depended upon the extrapolation of data gained from experiments which lasted no more than 30 minutes as well as an unusually high value for the TRM. Rimbert $(1959$, p. 39) used a field of $0.5 œ$ in similar experiments which lasted 8 days on a sample of Auvergne basalt. Extrapolation of her data yielded a time of the order of $10^{4}$ years for the VRM to become an appreciable fraction of the TRM.

The enormous differences in rate of acquisition of VRM implied by these experiments might represent intrinsic differences in the samples, but it is more likely that variations in the particulars of the experimental design and the necessity of extrapolating over many orders of magnitude of time are responsible for a large part of the disparity in magnetic stability. Thus it appears that the shape of the NRM-TRM curve is the best available 
indicator of the extent to which the original TRM has been modified by VRM.

Multidomain grains. The single-domain type of model appears incapable of explaining the NRM-TRM curves of some samples. For example, in a previous paper (Coe, 1967) reference was made to the peculiar behavior of the $1915 \mathrm{Mt}$. Lassen dacite flow. Eleven samples tested were magnetically rather soft and yielded NRM-TRM curves of such variable shape (mostly concave up or $S$-shaped) that the paleo-intensity determined by fitting a line to the best straight segment of each curve might have varied by a factor of two or three, depending on one's particular interpretation. The paleo-intensities determined from the straight lines through only the initial and final points of all the curves, however, yielded an average of $0.53 \pm .06 œ$, in remarkable agreement with the known figure of $0.54 \pm .02 œ$ at Mt. Lassen in 1915.

This result could be explained by the coincidental cooperation of independent non-ideal mechanisms, but a more likely interpretation would involve one mechanism which changes the temperature spectrum of the remanence without affecting the total NRM. A similar mechanism, or perhaps the same one magnified by some effect of ac demagnetization, is also needed to explain the results of the experiments described above in which Thellier's method and ac demagnetization were combined.

In cases such as these at least part of the TRM may arise from a mechanism involving the displacement of domain walls past potential barriers within multidomain grains. In all theories based on this sort of model it is the combined effect of the external field and the self-demagnetizing field within each grain which causes the walls to move (Néel, 1956; Everitt, 1962; and Stacey, 1963). Because the demagnetizing field depends directly on the intensity of magnetization, the PTRM acquired in a particular temperature interval cannot be independent of the state of magnetization of the grain, and non-ideal results may be obtained from Thellier's method.

For instance, when a grain cools in an external field, the walls which were blocked at high temperatures ride high on the sides of the potential barriers because the demagnetizing field increases during cooling. Thermal demagnetization is only the exact reverse of the acquisition of TRM if it is done by heating in the same field and measuring the loss of remanence at each elevated temperature. In the usual method of demagnetization, however, the sample is cooled in zero external field. This enhances the effect of the grain demag. netizing field, so each domain wall rides higher on the potential barrier which pins it, and less thermal agitation (or less of a decrease of barrier height) is required for the wall to surmount the barrier. Thus the whole TRM spectrum is shifted to lower temperatures during thermal demagnetization.

Moreover, the possible effects of thermal fluctuations on the original TRM over a long period of time could be quite complex. As in the case of single-domain grains, the TRM acquired at the lowest temperatures would be expected to be initially stable if the field in which it was produced remained constant. As the remanence decreased due to the shifting of walls that were pinned at higher temperatures, however, the demagnetizing field would decrease, thereby increasing the effective field inside the grain. The walls pinned at lower 
temperatures would move, tending to restore the internal field to its previous value, so that the net effect would be a transfer of remanence from higher to lower blocking temperatures.

The above are only two of many possible non-ideal mechanisms which might occur in multidomain grains. They are only meant to be illustrative, and may not apply to the particular examples cited. Much more information (such as the domain and potential barrier configurations and the dependence of barrier height on temperature) is needed, and considerable advances in theory must be made, before specific mechanisms can be identified in actual samples.

Effect of disparities in cooling rate. The length of time over which a lava flow cools while it acquires a natural TRM is sometimes longer by as much as a factor of $10^{4}$ than the time in which artificial TRM was produced in the lab. If two PTRM's are produced in two identical, initially unmagnetized assemblages of single-domain grains by cooling in a weak field from 400 to $20^{\circ} \mathrm{C}$, the first one within $t$ sec and the second within $t \times 10^{4} \mathrm{sec}$, the second PTRM will be greater chiefly because grains with longer relaxation times will be mag. netized. If the number of grains with a given volume $v$ and coercivity $H_{c}$ is uniformly distributed in the $v \cdot H_{c}$ plane, then application of results derived by Néel $(1949, \mathrm{p} .131)$ shows that the second PTRM will exceed the first by about $20 \%$.

However, the case of a total TRM, and therefore lavas, is different. At any temperature during cooling the grains with longer relaxation times have already been magnetized. Thus increasing the length of time of cooling can increase the total TRM only by increasing the proportion of grains aligned, and hence the magnitude of the effect is probably much less than the $20 \%$ for the PTRM, which can be increased by aligning grains that were previously randomly oriented. It is difficult to determine directly because the integration of a com. plicated expression which contains many quantities that cannot be easily measured is required.

If the effect is significant samples from the center of a thick flow should indicate systematically higher paleo-intensities than samples from the margins. If several thick flows with differing magnetic mineralogies are found which give reliable and constant paleointensities from center to margin, than differences in cooling rate are probably not critical in the acquisition of a total TRM. No systematic variations can be demonstrated from my results, but none of the data is ideally suited for this test.

Nonlinearity of acquisition of TRM. Another factor which could change the experimentally determined NRM-TRM curve from the ideal straight line is the nonlinearity of TRM with field. Assuming the nonlinearity to be always in the direction of saturation, Fig. 7 depicts some possible effects for the cases $F_{a}>F_{e}$ and $F_{a}<F_{e}$.

If the degree of nonlinearity is the same for all PTRM segments, the NRM-TRM curve remains straight but is changed in slope from the ideal case. If nonlinearity is concentrated in a PTRM range, curvature results.

A graphical method to correct the paleo-intensities determined by Thellier's method for the effects of nonlinearity is illustrated in Fig. 8. First the relation between TRM and field must be determined; it is assumed, just as in Thellier's method, that the PTRM spectrum 


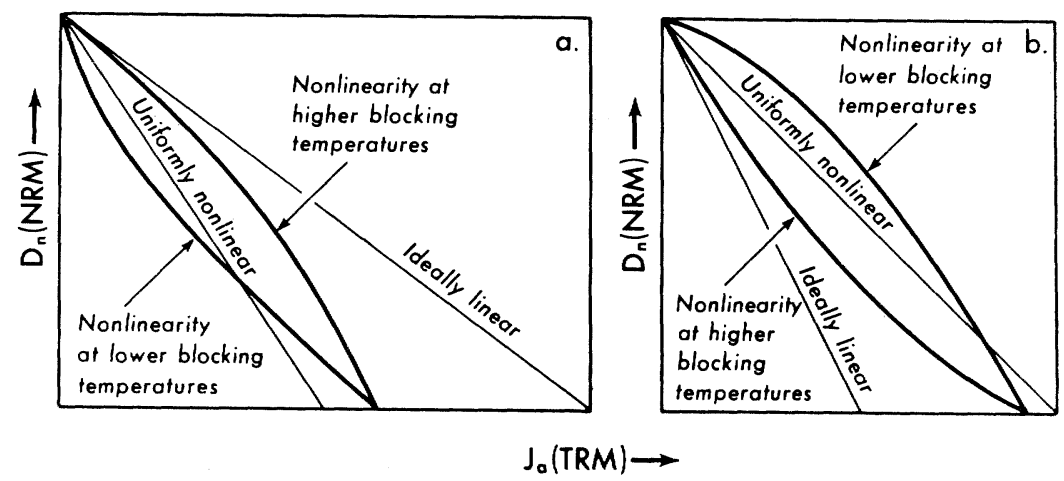

Fig. 7 Possible NRM-TRM curves for various relationships between TRM and applied field when (a) $F_{a}=(4 / 3) F_{e}$ and (b) $F_{a}=(1 / 2) F_{e}$.

of the rock undergoes no changes during the heating up to a temperature $T_{f} \leqslant T_{c}$. The apparent paleointensity determined according to (1) is equivalent to $F_{e}^{\prime}=\left(J_{e} / J_{a}\right) F_{a}$ of Fig. 8, where $J_{e}$ and $J_{a}$ are NRM and TRM produced in the ancient field $F_{e}$ and the artificial field $F_{a}$, respectively, during cooling from the elevated temperature $T_{f}$ to $T_{r}$. Thus $F_{e}^{\prime}$ must be corrected by an amount $\Delta F_{e}$ to the true value $F_{e}$, which corresponds to shifting from a point on the hypothetical linear magnetization curve to the true nonlinear one. The magnitude of $\Delta F_{e}$ is seen to depend not only on the degree of nonlinearity but also

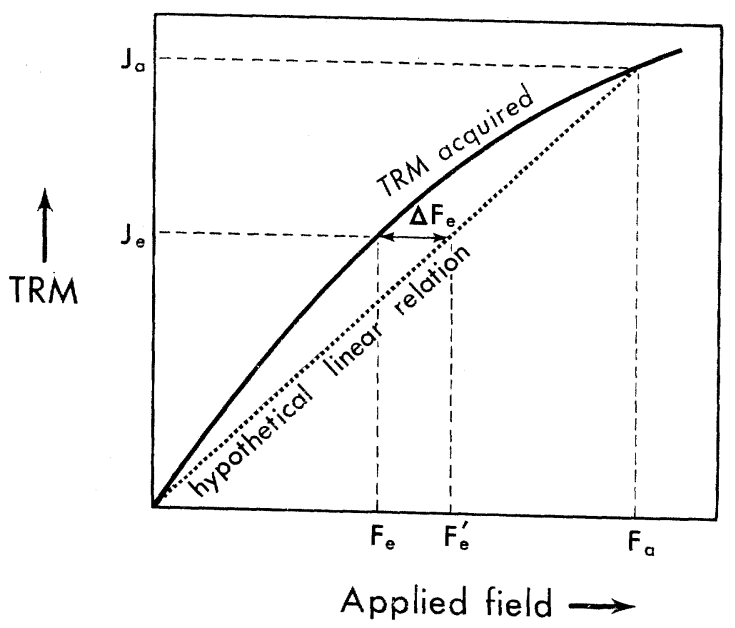

Fig. 8 A graphical method for determining the correction term $A F_{e}$ to the experimentally derived paleo-intensity $F_{e}{ }^{\prime}$ when the TRM acquired is not a linear function of the applied field.

on the difference between $F_{a}$ and $F_{e}$; if $F_{a}=F_{e}$, then $F_{e}^{\prime}=F_{e}$, and the correction is zero.

Curves of total TRM versus field were determined between 0.0 and $2.29 œ$ for 22 samples from 16 of the 18 volcanic units which were discussed previously (Coe, 1967). At $2.29 œ$ all samples but two deviated visibly from linearity toward saturation; i.e., the deviations are greater than the experimental scatter in reproducing a given point. This usually occurred around $0.5 œ$. Despite this considerable nonlinearity, however, the corrections $\Delta F_{e}$ were overshadowed greatly by the experimental uncertainties in $F_{e}^{\prime}$ and thus were not applied. (All 22 curves of TRM versus field can be found in my thesis (Coe, 1966)).

The errors caused by nonlinearity were negligible in this study only because none of the paleo-intensities estimated exceeded $0.50 œ$ by very much and because in most cases $F_{a}$ was not too much greater than $F_{e}^{\prime}$. If an apparent paleo-intensity of $1.0 œ$ had been determined for the most nonlinear sample, and if $F_{a}=0.5 œ$, then the true value would have been 
$1.1 œ-$ a correction of $10 \%$. Comparable errors due to neglecting nonlinearity would have resulted for several samples if $F_{e}$ had equalled $2.3 œ$ when $F_{a}=0.5 œ$.

Irreversible changes in TRM capacity. Perhaps the most common source of non-ideal behavior is irreversible changes of the TRM spectrum during the laboratory heating experiments. The alteration appears to depend more strongly on the maximum temperature reached rather than the length of time of heating; repetition of a heating step hardly ever produces significant differences in magnetization from the previous step. The detailed mechanisms of these changes are at present a matter of conjecture. Oxidation (or possibly reduction), ionic ordering (or disordering), exsolution of separate phases (or homogenization), annealing (or unannealing) and other phenomena may all contribute in various rocks.

It is important to detect these changes whether or not the exact mechanisms are known. A test devised by the Thelliers (1959) and recently used by Briden (1966) consists of repeating the determination of PTRM in a given temperature interval after the sample is exposed to each higher temperature. Significant, progressive changes in the PTRM acquired in this interval indicate that the rock is behaving non-ideally.

The weakness of this "PTRM test" is that it can never conclusively demonstrate the absence of changes of PTRM in temperature intervals above the reference interval, and hence it is impossible to determine entirely the changes in the TRM spectrum of a rock caused by heating. The best one can do is determine the PTRM spectrum from room temperature through the temperature of the last heating step for all temperatures that are used up to the Curie point. Because of the time-consuming nature of these experiments, however, a more useful procedure is to measure the greatest possible PTRM segment, that between room temperature and the temperature $T_{i}$ of the last heating step, after completing the step at the next higher temperature, $T_{i+1}$.

Fairly complete tests were conducted on 44 of the samples studied, while only intermittent checks of PTRM were performed on the remaining 51. In general the shapes of the NRM-TRM curves are reflected by the results of the PTRM tests, especially at higher temperatures where secondary components of magnetization are less important and where the test incorporates a more significant fraction of the total TRM. Increase of PTRM with increasing tomperature is the most common change, and accordingly the most common curvature is concave up. A few percent of the NRM-TRM curves, especially those concave down, were inconsistent with the PTRM tests and hence must have been affected by a different non-ideal mechanism.

In order to test the correlation more objectively and in greater detail, it was necessary to make two arbitrary definitions. Without launching into too many details (see Coe, 1966 for full definitions), a straight segment of an NRM-TRM curve is defined as one of reasonable length which consists of at least six points, none of which deviate from the best fitting straight line by more than $5 \%$ of the total TRM. Similarly, a significant variation in PTRM of a given interval is taken as one being greater than $5 \%$ of the total TRM.

According to these definitions, 34 of the 44 NRM-TRM curves adequately tested have straight segments, and 22 of these 34 samples show no significant PTRM changes in the 


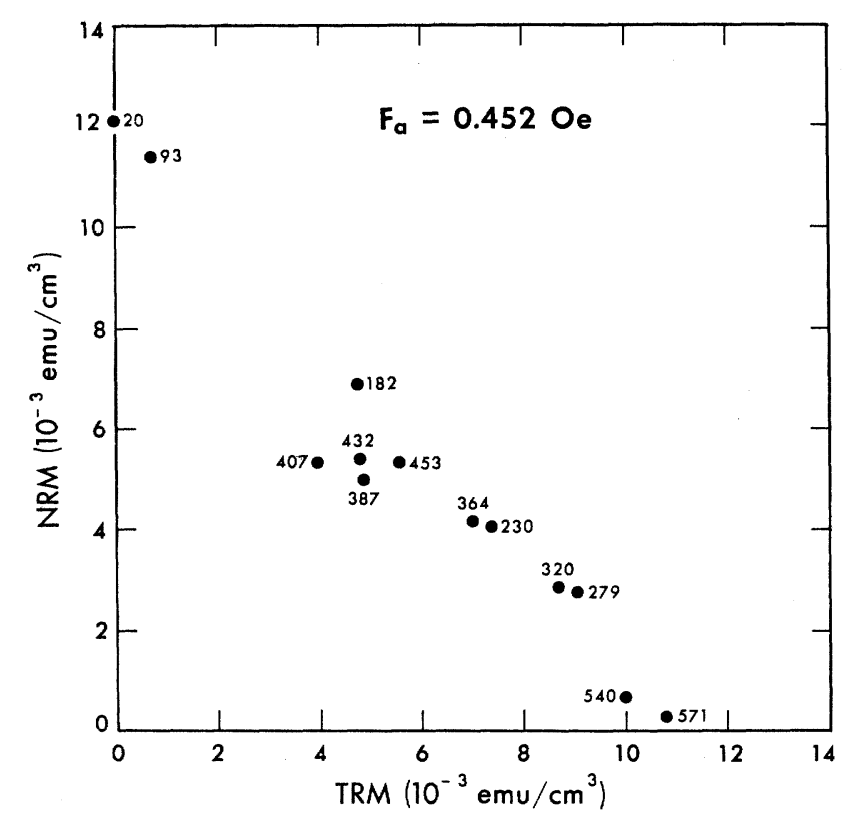

Fig. 9 The NRM-TRM curve of a Recent basalt which has a reversed component in the NRM and also acquires one in the TRM. The numbers associated with the points are the temperatures of the heating steps in ${ }^{\circ} \mathrm{C}$.

temperature interval corresponding to the straight line segment. Of the remaining twelve, seven changed by more than $5 \%$ during only a single heating step and two during two heating steps. The most extreme exception is shown in Fig. 9. This sample of Recent basalt from the Devil's Homestead Flow, Lava Beds National Monument, California (Coe, 1966 and 1967) had a reversed component between 279 and $432^{\circ} \mathrm{C}$ in the NRM and also acquired one in the laboratory TRM between $279^{\circ}$ and $407^{\circ} \mathrm{C}$. Thus nine of the thirteen points of the NRM-TRM curve lie on a straight segment, but the PTRM test shows changes of up to $50 \%$. Although in this case the straight segment is obviously suspect because the curve retraces itself with increasing temperature, in the other few cases where significant PTRM changes occur within a straight segment the straightness is fortuitous and does not imply a reliable paleo-intensity.

Even more meaningful is the fact that the deviations of points from the straight segment at high temperature are almost always qualitatively documented by the PTRM test. Only two of the 34 samples were exceptions to this generalization. An example of this, as well as its usefulness in helping to define the pertinent straight segment of an NRM-TRM curve, has been discussed previously (Coe, 1967, figure 4 and text).

A natural further step is to use the results of the PTRM test to apply corrections to the points of the NRM-TRM curves. Briden (1966) appears to have been the first to try this. He defined a factor $C$ equal to the PTRM in the range 300 to $100^{\circ} \mathrm{C}$ measured after heating to $600^{\circ} \mathrm{C}$ divided by the average value of the PTRM in the same range measured after heating to 300,400 and $500^{\circ} \mathrm{C}$. Corrected values of the PTRM in the ranges 600 to $400^{\circ} \mathrm{C}$, 
600 to $500^{\circ} \mathrm{C}$ and 600 to $550^{\circ} \mathrm{C}$ were then obtained by dividing the experimentally determined PTRM's by $C$, bringing the high-temperature results into "broad consistency" with those of lower temperatures. However, the definition of the factor $C$ is rather arbitrary, as Briden is the first to acknowledge. It is difficult to justify belief in results which have been arbitraily adjusted unless the same adjustments bring strikingly increased consistency when applied to a variety of different rocks which have undergone irreversible physico-chemical changes.

I have independently tried similar corrections. Two schemes, perhaps somewhat simpler, but equally arbitrary, were used, based on the assumption that either (a) all PTRM segments behave the same may, so that changes of PTRM in a low temperature interval signify proportional changes in every higher temperature interval or (b) changes are confined to the PTRM residing in the interval up to but not above the highest temperature reached. Neither assumption holds rigorously for all the samples, although (b) appears to be the better of the two. The method of correction based on (b) yielded in a few cases corrected NRM-TRM curves which were strikingly straighter than the originals, but in most there was little improvement, and in a few instances the curvature became even more pronouned. The actual behavior of the rocks seems to be too complicated and varied for the use of a single, quantitative correction scheme based on reasonable physical assumptions.

\section{Diagnostic Tests}

Thellier's method is so time-consuming that it is important to search for diagnostic tests which predict whether or not a sample will behave ideally. At present no test has been developed which meets the twofold requirements of quickness and reliability.

Reversiblity of the saturation magnetization versus temperature curves. Measurement of the saturation magnetization $\left(J_{s}\right)$ as a function of temperature $(T)$ is a common experiment for determining Curie points. In addition, the degree of irreversibility of the processthat is, the degree to which the heating and cooling $J_{s}-T$ curves do not coincide-is often used to assess qualitatively the extent of alterations induced by the heating of the magnetic minerals.

Curves of this type were determined for 21 cores from 14 different volcanic units (including basalts, andesites, dacites, pumice, agglutinates and baked sediments) with the equipment of the Rock Magnetics Laboratory, U.S. Geological Survey, Menlo Park, California (Doell and Cox, 1966). All samples were tested in a purified nitrogen atmosphere such as was used for the Thellier experiments (Coe, 1967). In addition, some duplicates were tested in a vacuum of about $10^{-8}$ to $10^{-7} \mathrm{~atm}$, and one other was tested in air. Despite the great range in partial partial pressure of oxygen, samples of the same core behaved rather similarly in all atmospheric environments. All $J_{s}-T$ curves can be found in my thesis (Coe, 1966).

Because $J_{s}$ is not sensitive to as many factors as TRM (such as impurity and dislocation concentration or grain-size distribution), one might expect the $J_{s}-T$ curves not to reflect all the subtleties of the NRM-TRM curves. Moreover, because rocks sometimes contain more than one magnetic phase, the possibility exists that changes in $J_{s}$ may occur in a phase which carries very little TRM while the other phases remain unaltered, so that a sample 
with an ideal, straight NRM-TRM curve might also have an irreversible $J_{s}-T$ curve.

Experiments confirm such expectations; comparison of the two types of curves shows that in general there is no obvious correlation between them, although in some specific instances deviations from a straight line in the NRM-TRM curve of a sample at a given temperature $T_{f}$ is matched by increased irreversibility in the $J_{s}-T$ curve when the maximum temperature is raised above $T_{f}$. There are just as many instances, however, where
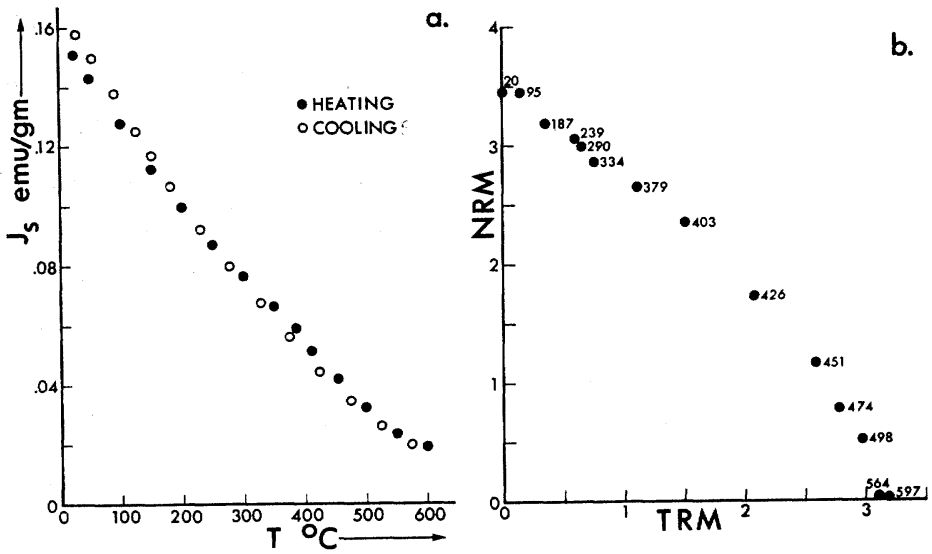

Fig. 10 (a) The $J_{s}-T$ curve for a sample of the Pleistocene Skull Cave basalt (Coe, 1967, table 1). The shape is unusual (concave up), but the heating and cooling curves are coincident. The applied field was $5500 œ$.

(b) The NRM-TRM curve for a sample from the same core. The curve deviates markedly from a straight line after $403^{\circ} \mathrm{C}$, and consistent changes in the TRM spectrum were detected in the PTRM test, despite the fact that no significant changes in $J_{s}$ occur during heating under the same conditions. $F_{a}=0.452 œ$. The figure is labelled in the same way as figure 4 .

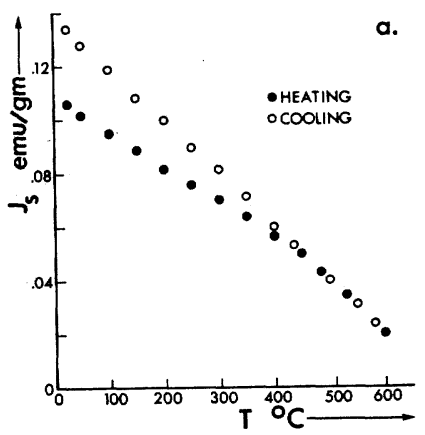

a.

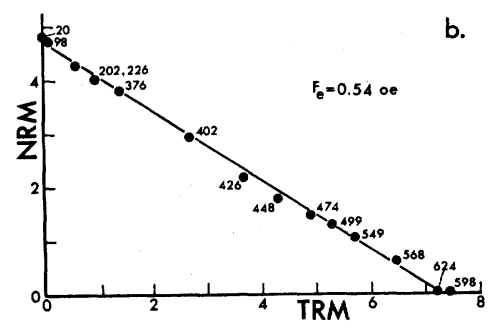

Fig. 11 (a) A rather irreversible $J_{s}-T$ curve for a sample of the Recent (?) Schonchin Butte agglutinate (Coe, 1967, table 1). The applied field was $9500 œ$.

(b) The NRM-TRM curve for a sample of the same core. It is reasonably straight despite the irrversible changes in $J_{s}$ which occur during heating under the same conditions. $F_{a}=0.834 œ$. The figure is labelled in the same way as figure 4 . 
deviations from ideality are manifested in the NRM-TRM curves (and in the PTRM tests), but not in the $J_{s}-T$ curves, as the example in Fig. 10 demonstrates. Likewise, samples which have straight NRM-TRM curves may show $J_{s}-T$ curves which are essentially reversible or, as shown in Fig. 11, quite irreversible. Finally, the $J_{s}-T$ measurements give no inkling of self-reversing tendencies in a rock, nor of the complicated NRM-TRM curves which often result from this behavior.

The only generalizations that can be made from this work are weak ones. The volcanic rocks which were most satisfactory in the Thellier experiments have high Curie points (greater than $550^{\circ} \mathrm{C}$ ) and regular $J_{s}-T$ curves with no pronounced lower Curie points. Some others that were failures, however, also had these very same characteristics. Conversely, little success was obtained with volcanic rocks which have low Curie temperatures and initially steep or irregular $J_{s}-T$ curves. The highest percentage of success was with baked sediments, regardless of whether the $J_{s}-T$ curves were fairly irreversible or reversible, initially steep and irregular or quite regular in shape. Thus the $J_{s}-T$ curves seem sometimes to provide relevant information, but not with sufficient reliability to be used with confidence for diagnostic purposes.

Irreversible changes in weak-field susceptibility. The weak-field susceptibility of fourteen specimens from seven volcanic units (four basalts, two agglutinates and one baked sediment) were measured at room temperature with an ac bridge after the completion of each heating step in Thellier's method. However, the irreversible changes of this quantity show very little correlation with those of the TRM spectrum. In general the susceptibility increased as heating progressed to higher temperatures, but the changes bore no regular relation to the deviations of the NRM-TRM curves from straight lines. In fact, abrupt changes in susceptibility are often not accompanied by significant changes in slope of the NRM-TRM curve, and vice versa.

Degree of linearity of TRM with field. Nonlinearity in the acquisition of TRM has been discussed above as a possible source of non-ideal behavior. It turns out, however, that the samples for which the TRM is most linear with field are among the least successful in Thellier's method. Most, but not all, of these tend also to have low Curie points and irregular $J_{s}-T$ curves. The typically nonlinear samples (over $70 \%$ of those tested) include both those that were best in Thellier's method and those that were worst. Thus, although the degree of linearity of a rock appears in some cases to yield useful information, it is not a useful diagnostic test. It is not discriminating enough, and, because the production of several total TRM's is involved, it is too time-consuming.

Comparison of the ac demagnetization curves of the NRM and total TRM. A method which was hardly used in these studies, but which may be a sufficient test of suitability for Thellier's method, is the comparison of the normalized ac demagnetization curves of the NRM and of the total TRM produced artificially in the laboratory. Coincidence of the curves demonstrates both the absence of changes in the coercivity spectrum (and thus perhaps in the blocking temperature spectrum as well) caused by heating in the laboratory and also the absence of significant secondary components of magnetization or spontaneous decay of 
the NRM.

Momose (1963) published fourteen such comparisons with matching comparisons of the thermal demagnetization curves of the NRM and total TRM for the same samples. In only one of these do the ac demagnetization curves clearly coincide, and in this sample the thermal curves do coincide also. There were two borderline cases where the ac curves nearly coincided, and in one of these the thermal curves were identical and in the other not. However, the thermal curves of five of the eleven remaining samples with dissimilar ac curves were coincident. Thus the ac demagnetization test is too stringent; it is not a necessary condition for success in the thermal experiments. This conclusion is supported by the two samples (baked sediments) tested in this study. Both behaved reliably in the Thellier experiments, but the ac demagnetization curves of the NRM and the total TRM coincided in one case and did not in the other.

This test is overly stringent for another reason; it requires heating to the Curie point in order to produce the total TRM. Samples which give reliable paleo-intensities in the Thellier experiments at low temperatures, but which undergo physico-chemical changes which affect the TRM spectrum at high temperatures, would not give identical ac demagnetization curves.

The ultimate goal of the diagnostic tests-petrologic determination of paleo-intensities. There remains a great deal of work to be done in formulating reliable diagnostic tests. What is needed is a much greater knowledge of the physico-chemical changes which affect the TRM of volcanic rocks.

One avenue of approach is to study both the petrology of the magnetic minerals of suites of volcanic rocks before and after detailed Thellier experiments and the $J_{s}-T$ curves of the same rocks. A brief attempt of this was made. 46 polished sections of selected samples were examined in reflected light, but no definite conclusions were reached. Nonetheless, I feel that significant progress might be made by someone expert in the study of iron-titanium minerals who also has an interest in rock magnetism.

The ultimate goal of such a study would be to advance our knowledge to the stage where a quick examination of the magnetic mineralogy and a single measurement of the NRM are all that is required to determine the strength of the ancient field which produced the original TRM. This stage is a long way off, however, and may never be reached except. in fantasy.

\section{Quicker Methods for Determinimg Paleo-intensities}

Even if diagnostic tests never achieve this ultimate goal, it is probable that someday we will be able to predict with reasonable certainty whether a rock will behave ideally. When we can, there will no longer be the need to use the complete method of Thellier. Until then, however, the question of the effectiveness of these abbreviated methods compared to Thellier's method must be considered.

Thellier's full stepwise method versus the simple comparison of NRM to total TRM. Thellier's method, although it involves more time and effort, is preferable to the direct 
comparison of the NRM to the total TRM acquired in a known field when accurate determinations of individual paleo-intensities are of importance. Even though many fewer samples can be studied in a given time, the additional information gained usually more than com. pensates.

For instance, the mean paleo-intensity derived by Thellier's method for the Alturas baked sediment (Coe, 1967, table 1 ) is $0.153 \pm .007 œ$, whereas the ratios of NRM to total TRM yield a mean of $0.141 \pm .033 œ$. The second value is probably too low, and its uncertainty is five times greater than that of the first. The analogous values for the Hat Creek basalt are $0.223 \pm .009 œ$ and $0.24 \pm .06 œ$. The increased uncertainty in the second value arises mostly because an aberrant paleo-intensity of $0.36 œ$ for one sample was retained, whereas it was not included in the first value because the NRM-TRM curve revealed extreme non-ideal behavior. A convincing case is necessary to justify such discarding of data, and the information required is often best provided by Thellier's method.

Nevertheless, Thellier's method may not be necessary in determining the mean geomagnetic intensity of a period in the geologic past. As long as systematic effects such as VRM and spontaneous decay are not large, the many other disturbing effects may possibly average out over a suitably heterogeneous suite of volcanic units. In support of this, the average paleo-intensity for the Recent found in this study by selecting the best data according to Thellier's method was $0.43 \pm .12 œ$ (Coe, 1967), while the uncritical average determined by the ratio of NRM to total TRM for all the samples is $0.43 \pm .15 œ$. For the time from about $10^{4}$ to $10^{7}$ years ago the analogous averages are $0.30 \pm .08 œ$ and $0.36 \pm .17 œ$, respectively. These comparisons of averages are not so significant as if they involved hundreds of different rock units, but their rough agreement suggests the possibility that this cruder method may be of great usefulness in this type of study.

Finally, for some rocks which fail in Thellier's method the comparison of NRM to total TRM may be a better method. This was certainly the case for the Mt. Lassen dacite discussed above; the simple comparison yields an average field intensity remarkably close to the known value, whereas there are no straight segments on the NRM-TRM curves which correspond to nearly such a good estimate. This may be a coincidence, but it may equally well demonstrate a property of non-ideal TRM of some multidomain grains.

Comparison of the thermal demagnetization curves of the NRM and the total TRM. A method almost equivalent to Thellier's is the comparison of the normalized thermal demagnetization curves of the NRM and a total TRM produced in a known field. It involves the same amount of work (though less computation) when measurements are made with conventional apparatus at room temperature. If the measurements are made in a special magnetometer at elevated temperatures during demagnetization, however, this method saves considerable time (Wilson, 1961 and 1962: Smith, 1967). The main disadvantage is that useful information may be destroyed when heating to the Curie point in order to produce the total TRM. Changes in the TRM spectrum may obscure ideal behavior at low temperatures which would have been utilized by Thellier's stepwise method.

This deficiency could be minimized, however, by using the PTRM test to determine 
where such irreversible changes in the TRM spectrum take place. If no changes were found to occur until a certain temperature $T_{f}<T_{c}$ had been exceeded, then the above method could be modified by comparing thermal demagnetization curves of a PTRM restricted in range from room temperature to $T_{f}$ instead of a total TRM. Such a method would retain the benefits of Thellier's method, yet would be considerably quicker.

\section{Acknowledgments}

I would like to thank Miss Beth Hurlbut, Mr. Ron Merrill, Dr. Allan Cox, Dr. Sherman Grommé, Dr. Richard Doell, and especially Prof. John Verhoogen, my thesis advisor, for their substantial contributions of time, effort and equipment to this project. In addition I gratefully acknowledge fellowships and research assistantships from the National Science Foundation (N.S.F. Grant Gp-5047) and the Pan American Petroleum Foundation during which most of the work was done at the University of California at Berkeley, and a postdoctoral research fellowship (funded by the Air Force Office of Scientific Research and administered by the National Academy of Sciences-National Research Council) at the Australian National University, Canberra during the final writing.

\section{References}

Briden, J.C., Estimates of direction and intensity of the paleo-magnetic field from the Mugga Mugga Porphyry, Australia, Geophys. J., 11, 267-278, 1966.

Coe, R.S., Paleo-intensities of the geomagnetic field determined from Tertiary and Quaternary rocks, Ph. D. Thesis, Dept. of Geol. and Geophys., Univ. of Calif., Berkeley, Calif., 1966.

Coe, R.S., Paleo-intensities of the earth's magnetic field determined from Tertiary and Quaternary rocks, J. Geophys. Res., 72, 3247-3262, 1967.

Cox, Allan, Anomalous remanent magnetization of basalt, U.S. Geol. Surv. Bull. 1083-E, 131-160, 1961.

Cox, Allan, R.R. Doell and G.B. Dalrymple, Reversals of the earth's magnetic field, Science, 144, 15371543, 1964.

Cox, Allan, D.M. Hopkins and G.B. Dalrymple, Geomagnetic polarity epochs: Pribilof Islands, Geol. Soc. Amer. Bull., 77, 883-910, 1966.

Doell, R.R. and Allan Cox, Recording magnetic balance, in Methods in Paleomagnetism, edited by Collinson, D.W., K.M. Creer and S.K. Runcorn, Elsevier Pub. Co', Amsterdam, 1966.

Doell, R.R. and G.B. Dalrymple, Geomagnetic polarity epochs : a new polarity event and the age of the Brunhes-Matuyama boundary, Science, 152, 1060-1061, 1966.

Everitt, C.W.F., Thermoremanent magnetization. III. Theory of multidomain grains, Phil. Mag., 7, 599616, 1962.

Momose, K., Studies on the variations of the earth's magnetic field during Pliocene time, Bull. Earthquake Res. Inst., 37, 487-534, 1963.

Nagata, T., Rock Magnetism, Maruzen, Tokyo, 1961.

Nagata, T., Y. Arai and K. Momose, Secular variation of the geomagnetic total force during the last 5000 years, J. Geophys. Res., 68, 5277-5281, 1963.

Néel, L., Théorie du trainage magnétique des ferromagnétiques en grains fins avec applications aux terres cuites, Ann. Géophys., 5, 99-148, 1949.

Néel, L., Some theoretical aspects of rock magnetism, Phil. Mag. Supp. Adv. Phys., 4, 191-243, 1955.

Ozima, M. and M. Ozima, Origin of thermoremanent magnetization, J. Geophys. Res., 70, 1363-1369, 1965. 
Rimbert, Francine, Contribution à l'étude de l'action de champs alternatifs sur les aimantations rémanentes des roches: applications géophysiques, Rev. Inst. Français Pétrole et Ann. Combustibles Liquides, 14, nos. 1 and 2, 75p., 1959.

Shimizu, Y., Magnetic viscosity of magnetite, J. Geomag. and Geoelect., 11, 125-138, 1960.

Smith, P.J., The intensity of the Tertiary geomagnetic field, Geophys. J., 12, 239-258, 1967.

Stacey, F.D., The physical theory of rock magnetism, Adv. Phys., 12, 45-133, 1963.

Stacey, F.D., Koenigsberger ratio and the nature of thermoremanence in igneous rocks, Earth and Plan. Sci. Letters, 2, 67-68, 1967.

Stoner, E.C., The demagnetizing factors for ellipsoids, Phil. Mag., 36, 803, 1945.

Thellier, E. and O. Thellier, Sur l'intensité du champ magnétique terrestre dans le passé historique et géologique, Ann. Géophys., 15, 285-376, 1959.

Verhoogen, J., The origin of thermoremanent magnetization, J. Geophys. Res., 64, 2441-2449, 1959.

Wilson, R.L., Paleomagnetism in Northern Ireland; part I, the thermal demagnetization of natural magnetic moments in rocks, Geophys. J., 5, 45-59, 1961.

Wilson, R.L., An instrument for measuring vector magnetization at high temperatures, Geophys. J., 7, 125-131, 1962. 